\title{
Familien- und Gleichstellungspolitik in Schweden - ein Vorbild für die Schweiz?
}

\section{Erkenntnisse der Forschungs- und Studienreise 2017 des Masters „Soziale Arbeit“ in Stockholm}

\author{
Lucia M. Lanfranconi, ${ }^{1}$ Simone Küng ${ }^{2}$
}

\begin{abstract}
Einführung
Der schwedische Wohlfahrtsstaat ist der typische sozialdemokratische Wohlfahrtsstaat nach Esping-Andersen (1990). Er basiert auf Vollbeschäftigung und der Teilnahme aller Bürgerinnen und Bürger am Arbeitsmarkt. Entsprechend verfolgt Schweden seit den 1970er Jahren eine Familienpolitik, die berufstätige Eltern unterstützt. Es wird von der Vorstellung einer gemeinschaftlichen Elternschaft ausgegangen. Die drei Pfeiler Individualbesteuerung, öffentliche Kinderbetreuung und Elternurlaubsversicherung sind wichtige Stützen. Die Familienpolitik steht zudem in enger Verbindung mit der öffentlichen Gleichstellungsstrategie, welche beispielsweise Unternehmen dazu verpflichtet, Gleichstellungspläne zu verfassen.

Die konstante und kohärente Familien- und Gleichstellungspolitik Schwedens hat zu einer hohen Erwerbsquote der Frauen und insbesondere der Mütter geführt: 83 Prozent der Mütter sind erwerbstätig. Die EU-Durchschnittsquote liegt bei nur 68 Prozent (OECD 2017a). ${ }^{3}$ Schweden übertrifft zudem die von der Europäischen Union geforderte Betreuungsquote von 33 Prozent für Kinder bis drei Jahre mit 56,7 Prozent (im Jahr 2014) deutlich (Eurostat 2017). ${ }^{4}$ Dennoch hat die Gleichstellungs- und Familienpolitik nicht zu einer gleichen Aufteilung der Betreuungs- und Hausarbeit zwischen Frauen und Männern geführt. Die Frauen tragen weiterhin die Hauptverantwortung für diese Arbeiten (OECD 2016: 3). Beobachten lässt sich ferner auch ein Geschlechterunterschied auf dem Arbeitsmarkt. Hier unterscheiden sich die strukturellen Positionen der Frauen von denjenigen der Männer - was z.B. zu Lohndifferenzen beiträgt. Der Arbeitsmarkt ist zudem weiterhin nach klassisch weiblichen und klassisch männlichen Berufffeldern segregiert (OECD 2017c: 2).
\end{abstract}

\footnotetext{
${ }^{1}$ Prof. Dr. Lucia M. Lanfranconi ist Dozentin und Projektleiterin an der Hochschule Luzern - Departement Soziale Arbeit: lucia.lanfranconi@hslu.ch.

${ }^{2}$ Prof. Simone Küng ist Dozentin an der Berner Fachhochschule - Departement Soziale Arbeit: simone.kueng@bfh.ch.

Unter Mitarbeit von: Gina Meyer, Cornelia Grob, Isabelle Scharnojahn, Samuel Nussbaum, Natascia Perrotto, Lesley Bamert, Silvio Imhof, Diego Pfammatter, Andrea Fröhlich, Simon Aebischer, Joel Erni, Aphrodita Zekiri und Lisa Zingg.

${ }^{3}$ Auch die Schweiz weist mit rund 78\% eine hohe Müttererwerbsquote auf (OECD 2017a). Ein grosser Unterschied zwischen den beiden Ländern wird jedoch bezüglich der Teilzeitquote deutlich: Diese liegt für Frauen in Schweden bei deutlich unter 20\% und in der Schweiz bei rund 45\% (Müller et. al 2017).

${ }^{4}$ In der Schweiz betrug der Anteil der formal betreuten Kinder im gleichen Alter im selben Jahr 29,8 Prozent (Eurostat 2017).
} 
Diese Ausgangssituation nehmen wir zum Anlass, uns in einer vergleichenden Perspektive genauer mit der schwedischen Familien- und Gleichstellungspolitik auseinander zu setzen. Wo kann Schweden als Vorbild für die Schweiz dienen, welches sind Best Practices? Wo gibt es jedoch auch erkennbare Widersprüche und Verbesserungsmöglichkeiten in Schweden? Welche Lernmöglichkeiten ergeben sich daraus für die Schweiz?

Dieser Artikel basiert zum einen auf dreizehn leitfadenbasierten ExpertInneninterviews in ausgewählten Institutionen in und um Stockholm. Es wurden mehrere Vertreterinnen des Departements Soziologie der Universität Stockholm, Personen aus zwei Unternehmen, der schwedischen Ombudsstelle, sowie VertreterInnen eines Sozialdienstes und solche eines Frauen-Integrationsprojektes befragt. Zum anderen wurden rund 25 leitfadengestützte Strasseninterviews mit Vätern und Müttern von Kleinkindern geführt. Alle diese Daten wurden im Rahmen der Forschungs- und Studienreise des konsekutiven Masterstudiengangs „Master of Science in Sozialer Arbeit" der Fachhochschulen Bern, Luzern, Zürich und St. Gallen vom 11. bis zum 15. September 2017 durch die 13 teilnehmenden Studierenden unter der Leitung von Prof. Dr. Lucia M. Lanfranconi und Prof. Simone Küng erhoben und ausgewertet. Die folgenden Abschnitte geben die Haupterkenntnisse wieder zu den Fragen: 1) Wo kann Schweden bezüglich Familien- und Gleichstellungspolitik als Vorbild für die Schweiz dienen? 2) Wo gibt es Widersprüche und Verbesserungsmöglichkeiten in Schweden? 3) Welche Visionen für die Zukunft werden genannt?

\section{„Auch ein Kleinkind hat ein Recht auf sozialen Kontakt ausserhalb der Familie“}

Die ersten Expertinneninterviews wurden am 11. September 2017 mit Ann-Zofie Duvander, Professorin der Soziologie und Demographie der Universität von Stockholm ${ }^{5}$ sowie drei Doktorandinnen desselben Departementes, die vorgängig den Film „Gleichstellen - eine Momentaufnahme“ (Lanfranconi/Lanfranconi 2016) zur betrieblichen Gleichstellungspolitik in der Schweiz geschaut haben, geführt.

\section{Schweden als Vorbild}

Die schwedische Familienpolitik ist seit den 1970er Jahren umfänglich darauf ausgerichtet, allen Bürgerinnen und Bürgern Zugang zum Arbeitsmarkt zu gewährleisten. Dieser innovative Ansatz führte dazu, dass die Aufgabe der Kinderbetreuung, die vorher hauptsächlich den Müttern oblag, im Sinne der gemeinsamen Elternschaft auf beide Elternteile verteilt wurde (Jönsson 2002: 176). Ebenfalls wurden die Angebote der Kinderbetreuung angepasst. Aus einer Analyse verschiedener Modelle in der Betreuungspolitik geht hervor, dass in Skandinavien die Kinderbetreuung hauptsächlich ausserhalb der Familie genutzt wird und egalitär geregelt ist. So äussert auch Duvander im Interview, dass man in Schweden - anders als teilweise im Schweizerischen Verständnis - davon ausgeht, dass es zum Wohle der Kinder sei, wenn diese fremdbetreut werden. Dementsprechend gibt es ein breites staatliches Angebot an Krippen,

\footnotetext{
${ }^{5}$ Ann-Zofie Duvander befasst sich vor allem mit der schwedischen sowie allgemein der nordischen Familienpolitik. Ihr Forschungsschwerpunkt liegt auf dem Elternurlaub mit Hauptaugenmerk auf dem von Vätern bezogenen Anteil.
} 
welches allen Familien zur Verfügung steht (Häusermann 2006: 4). Die Quote der Kinder im Alter von zwei bis fünf Jahren, welche eine Kinderkrippe besuchen, beträgt laut Duvander über 90 Prozent.

Die Vereinbarkeit von Berufstätigkeit und Familie für beide Elternteile wurde zu Beginn der 1970er Jahre noch durch weitere Massnahmen gefördert. So wurde der bestehende Elternurlaub für beide Elternteile erweitert, und ein neues Gesetz definierte das geteilte Sorgerecht nach einer Trennung der Eltern als Standard. Diese Massnahmen sollten beide Elternteile dazu ermuntern, am Arbeitsleben teilzunehmen und sich die unbezahlte Familienarbeit aufzuteilen. Dieses sogenannte „Earner-Carer-Modell“ wurde seitdem kontinuierlich ausgebaut, was dazu geführt hat, dass in Schweden sowohl die Frauenerwerbsquote als auch die Geburtenrate im europäischen Vergleich überdurchschnittlich hoch liegen (Duvander/Ferarrini 2013).

Der Elternurlaub beträgt derzeit 480 Tage pro Kind. 390 Tage davon werden einkommensabhängig entschädigt, die restlichen 90 Tage werden pauschal abgegolten. Um die Väter vermehrt zur Kinderbetreuung zu verpflichten, ist ein gewisser Anteil an Tagen pro Elternteil nicht übertragbar (sogenannte „Daddy-Months“). 2016 wurde dieser Anteil von 60 auf 90 Tage ein zweites Mal erhöht. Der von Vätern bezogene Elternurlaub hat nach jeder Erhöhung der „Daddy-Months“ jeweils markant zugenommen, so Duvander. Diese wissenschaftliche Evidenz habe die politische Entscheidung zur weiteren Erhöhung beeinflusst. Schweden kennt auch einen „Temporary Parental Benefit“, der es den Eltern erlaubt, im Krankheitsfall eines Kindes bis im Alter von zwölf Jahren bei der Arbeit zu fehlen. Pro Kind können dafür bis zu 120 Tage im Jahr eingesetzt werden (Duvander im Interview; MISSOC o.J.). Kontrollen diesbezüglich gibt es nur stichprobenartig, dennoch scheint die Leistung mehrheitlich nicht missbraucht zu werden. So beziehen gemäss Duvander Mütter 8.5 Tage und Väter 6.9 Tage im Schnitt pro Jahr.

Zudem erhalten Familien Kindergeld, Steuererleichterungen und haben einen garantierten Krippenplatz für jedes Kind, welcher umgerechnet nicht mehr als ca. 285.- Franken pro Monat, aber maximal vier Prozent des Einkommens kosten darf. Das zweite Kind erhält den Krippenplatz zu einem reduzierten Preis. Für alle weiteren Kinder einer Familie fallen keine weiteren Kosten mehr an. Damit haben auch Familien mit geringerem Einkommen und mehreren Kindern die Möglichkeit, familienexterne Kinderbetreuung zu nutzen (vgl. Duvander/Ferrarini 2013).

Im Gegensatz zum schwedischen Modell spricht Häusermann (2006) in Bezug auf die Schweiz von einer schwach entwickelten Familienpolitik. Die Betreuung der Kinder finde vorwiegend in den Familien statt. Familienexterne Kinderbetreuung ist in der Schweiz sehr teuer. So kostete die Ganztagesbetreuung von Kindern im Jahr 2011 rund 110 Franken pro Tag und Kind (vgl. Bundesrat 2015). Im internationalen Vergleich fielen finanzielle Transfers (Kinderzulagen etc.) eher tief aus, es gibt wenig bis kaum Mutterschutz, Elternurlaub oder Betreuungsinfrastruktur (Vgl. Bundesrat 2015). Die Kinderbetreuung werde nicht als Staatsaufgabe angesehen. Dass Frauen in der Schweiz unter den gegebenen Bedingungen noch immer oft zwischen Karriere und Kindern wählen müssen, führt dann auch dazu, dass vor allem hochqualifizierte Frauen auf Kinder verzichten und dass Mütter im internationalen Vergleich 
eine hohe Teilzeiterwerbsquote sowie eine eher tiefe Geburtenrate aufweisen (Häusermann 2006; Müller et al. 2017).

Auch die Gleichstellungspolitik ist in der Schweiz schwach entwickelt. Unternehmen werden bis anhin hauptsächlich durch staatlich finanzierte Projekte zu freiwilligen Massnahmen motiviert. Allerdings entscheiden Unternehmen dabei selbst über Inhalt und Durchführungsweise. Dabei besteht die Gefahr, dass diese Massnahmen nicht zur Gleichstellung, sondern „nur“ zur allgemeinen Mitarbeitendenzufriedenheit beitragen und eher Mitarbeitende mit höheren Machtpositionen betreffen. Bessere Aufstiegschancen für Frauen werden dadurch beispielsweise kaum geschaffen. Ein weiterer Kritikpunkt der freiwilligen Massnahmen der Schweiz ist, dass mehrheitlich nur für das Thema Gleichstellung sensibilisierte Firmen an Gleichstellungsprojekten partizipieren (Lanfranconi et al. 2017).

Bezüglich des Elternurlaubes kann die Schweiz mit 14 Wochen Mutterschaftsurlaub, entgegen der 96 Wochen Elternurlaub in Schweden, kaum mithalten. Einen gesetzlich vorgeschriebenen Vaterschaftsurlaub gibt es in der Schweiz bisher nicht (Müller et al. 2017). Eine Volksinitiative für einen gesetzlich verankerten vierwöchigen Vaterschaftsurlaub wurde 2016 erfolgreich lanciert, wird vom Bundesrat allerdings zur Ablehnung empfohlen. Stattdessen setzt der Bundesrat die Priorität bei der Förderung der familienergänzenden Kinderbetreuung (Bundesrat 2017). Auch eine Garantie für die Mutter, bei der Wiederaufnahme der Arbeitstätigkeit dieselbe Position zu besetzen wie vor dem Mutterschaftsurlaub, wie dies in Schweden vorhanden ist, ist nicht gegeben.

So zeigten sich die Studentinnen der Stockholmer Universität nach der Vorführung des Schweizer Films „Gleichstellen - eine Momentaufnahme“ (Lanfranconi/Lanfranconi 2016) sehr verwundert darüber, wie schnell sich Arbeitnehmende - vor allem Mütter - mit den Entscheiden und Vorgaben der Arbeitgebenden zufriedengeben, selbst wenn diese nicht in ihrem Sinne sind.

\section{Verbesserungspotenzial in Schweden}

So fortgeschritten das schwedische System scheint, auch dort besteht Veränderungs- und Verbesserungsbedarf. So stellte sich vor allem auch uns mit Schweizer Hintergrund die Frage, inwiefern in Schweden Wahlfreiheit besteht, wenn ein überwiegender Teil der Mütter Vollzeit arbeitet und sich rechtfertigen muss, wenn sie dies nicht tut.

Laut den befragten Doktorandinnen ist trotz der vorbildlichen Situation in Schweden in der Gleichstellung noch Verbesserungspotenzial vorhanden. So würde trotz der grosszügigen Möglichkeit des Elternurlaubes die Beanspruchung der Väter kaum über die an sie gebundenen 90 Tage hinausgehen. Daher werden die „Daddy-Months“ von den Doktorandinnen als effektivstes Mittel für die Einbindung der Männer in die Familienarbeit genannt. In Schweden wird derzeit ungefähr ein Viertel des Elternurlaubes von Vätern bezogen (Duvander/Andersson 2006). Die Diskrepanz in der Beanspruchung habe damit zu tun, dass immer noch mehr Männer Vollzeit beschäftigt sind als Frauen. Den Doktorandinnen nach ist dies hauptsächlich auf die Beschäftigungsart zurückzuführen. Während Männer überwiegend Vollzeit und in der Privatwirtschaft und in Kleinbetrieben tätig seien, würden Frauen eher im öffentlichen Sektor arbeiten, welcher Teilzeitarbeit fördert und geringere Löhne kennt. Zudem zeige sich, dass die Mehrheit der Führungstätigkeiten eher nicht mit reduzierten Pensen 
vereinbar sei. Beobachtbar sei aber, dass Mütter nach dem Mutterschaftsurlaub mit einer grossen Selbstverständlichkeit an den Arbeitsplatz in die alte Jobposition zurückkehren - ganz im Gegenteil zur Schweiz (Lanfranconi et al. 2017).

Zusammenfassend lässt sich festhalten: Während in der Schweiz die Kinderbetreuung noch immer als tendenziell private Angelegenheit der Mütter/Eltern erachtet wird und ein formales Kinderbetreuungsangebot mit hohen Kosten verbunden ist, erachtet Schweden die Kinderbetreuung als (Mit-)Aufgabe des Staates und geht davon aus, dass die professionelle Fremdbetreuung der Kinder und der Kontakt mit Gleichaltrigen zu deren Wohl beitrage. In der Schweiz müssen sich Mütter oft noch zwischen Karriere und Mutterschaft entscheiden oder arbeiten Teilzeit, wohingegen es in Schweden zur Norm geworden ist, dass eine Mutter Vollzeit arbeitet. Auch hier stellt sich aber die Frage, inwiefern Wahlfreiheit besteht. Es zeigt sich eindrücklich, wie stark die Prämissen darüber, was das Wohl des Kindes (externe Kinderbetreuung wünschenswert oder zu vermeiden) und wer für die Kinderbetreuung verantwortlich ist (Staat oder Familie), die Familienpolitik und die gelebten Realitäten in den Familien beeinflussen. Dies heisst aber auch, dass man solche Prämissen nicht als gegeben, sondern als diskutierbar anschauen darf. Nur so können innovative Ideen entstehen.

\section{"Geschlechtergleichstellung beginnt beim CEO"}

Schweden ist bekannt für die fortschrittliche Politik im Bereich der Geschlechtergleichstellung. Um einen konkreten Eindruck zu bekommen, wie die Umsetzung in der Praxis funktioniert, wurden die zwei Unternehmen Dedicare und Kungsleden besucht. Beide Unternehmen wurden aufgrund ihrer vorbildlichen Geschlechterverteilung in Leitungsfunktionen im AllBright-Rapport 2016 aufgeführt (AllBright 2016: 5). Am 12. September 2017 wurde je ein ExpertInneninterview durchgeführt, in Dedicare mit Frau Lia Sandström (CFO) und Herrn Stig Engcrantz (CEO) und in Kungsleden mit Frau Ylva Sarby Westman (Deputy CEO und Chief Investment Officer) und Frau Maria Sandell (Sustainability Manager).

\section{Kompetenzen als zentrales Anstellungskriterium}

Das 2009 in Kraft getretene Antidiskriminierungsgesetz, welches das bisherige Gesetz zur Gleichstellung von Frau und Mann ersetzt hat, fordert die Unternehmen dazu auf, aktiv die Gleichstellung von Frau und Mann zu fördern. Arbeitgebende mit mehr als 25 Mitarbeitenden sind demnach dazu verpflichtet, einen Massnahmenplan zur Gleichstellung zu erstellen (Schär Moser/Strub 2011: 29). Solche Gesetze brauche es, meint Westman von Kungsleden im Interview. Dennoch seien sie keine Garantie für Geschlechtergerechtigkeit in einem Unternehmen. Diese beginne nämlich beim CEO. Wenn auf Managementebene der Wille und die Bemühung bestehen, Gleichstellung zwischen Frau und Mann zu erreichen, sende dies ein bedeutendes Signal an alle Angestellten. In Kungsleden manifestiert sich dies im Vorleben einer Inklusionskultur. Mittlerweile geht es, wie Westman ausführt, nicht mehr nur um Frau und Mann, sondern um Diversität generell. Menschen verschiedener Geschlechter und Ethnien, Alter und Fachrichtungen sollten sich in Kungsleden wohlfühlen. Denn nur wo verschieden und vielfältig gedacht werde, könne Kreativität entstehen. Auch Engcrantz von De- 
dicare sieht das Thema nicht so eng eingegrenzt und unterstreicht die Wichtigkeit, alle Menschen gleich zu behandeln, egal welches Geschlecht, Alter, Hautfarbe oder sexuelle Orientierung sie haben. Es gehe in erster Linie um die Kompetenzen, welche die Angestellten mitbringen. So habe Dedicare vor kurzem eine schwangere Frau eingestellt, im Wissen, dass sie bald für einige Zeit ausfallen wird, dies, weil sie für die Stelle enorm geeignet sei und man sie auch nach dem Elternurlaub behalten wolle. Es werde langfristig gedacht.

Elternurlaub in Schweden fällt länger aus als in der Schweiz und führt dazu, dass Angestellte, je nach Aufteilung des Elternurlaubs, bis zu 420 Tage fehlen (MISSOC o.J.). Kungsleden und Dedicare sehen dies kaum als Problem. Da der Staat 80 Prozent des Lohnes übernimmt, bleibe genügend Geld, um eine Stellvertretung einzustellen. Gemäss Engcrantz kann dies sogar ein Vorteil sein, denn neue Angestellte können immer auch wichtige Inputs in das Unternehmen bringen. Allgemein hänge der Umgang mit dem Elternurlaub vor allem von der Haltung des Unternehmens ab, beteuert Engcrantz. Westman und Sandell schätzen, dass Frauen und Männer in Kungsleden je etwa gleich viel Elternurlaub beantragen und es somit für das Unternehmen keine Rolle spiele, ob eine Frau oder ein Mann eingestellt werde. Der lange Elternurlaub wird nie in Frage gestellt und die Sicherheit der Angestellten, dass sie nach dem Elternurlaub die gleiche Stelle zurückbekommen, wird als essenziell angesehen.

\section{Fokus auf Chancen und nicht auf Probleme}

Hinter dem grosszügigen Elternurlaub verstecken sich dennoch Stolpersteine. So kann eine ungleichmässige Aufteilung dessen eine Herausforderung darstellen. Obwohl bereits viele junge Väter vier bis sechs Monate Elternurlaub nehmen, sind es gemäss Engcrantz doch noch häufiger die Mütter, welche über längere Zeit von der Arbeit wegbleiben oder den Elternurlaub in Teilzeitform nutzen. Solche Unterschiede können sich negativ auf die Geschlechtergleichstellung auswirken. Bei Kungsleden wiederum sind im Bereich des Elternurlaubs keine Schwierigkeiten zu erkennen. Westman ergänzt aber, dass die Situation auf dem Land oder in Unternehmen mit nicht oder schlechter ausgebildetem Personal ganz anders aussehen könnte. Von geschlechtsbezogener beruflicher Segregation im Bewerbungsprozess bleibt aber auch Kungsleden nicht verschont, was sich darin äussert, dass sich für gewisse Bereiche mehr Männer und für andere mehr Frauen bewerben. Dennoch könne man für jeden Bereich kompetente qualifizierte Frauen finden, so Westman.

Obschon vieles in Sachen Geschlechtergleichstellung in Schweden gut läuft, sehen die beiden Unternehmen an einigen Stellen noch Verbesserungsbedarf. Für Engcrantz wäre ein erster wichtiger Schritt, dass Bewerbungsunterlagen ohne Angaben des Geschlechtes, des Alters oder der Herkunft eingeführt werden, sodass nur auf die Kompetenzen einer Person fokussiert wird. Gemäss Westman sollen des Weiteren staatliche Betriebe mit gutem Beispiel vorangehen, um so skeptischen Personen aufzuzeigen, dass Geschlechtergleichheit möglich ist.

\section{Und die Schweiz?}

Im Vergleich zur Schweiz ist Geschlechtergleichstellung sowohl in den beiden besuchten Unternehmen als auch in der schwedischen Gesetzgebung viel fester verankert und wird im Alltag gelebt. Damit hierzulande eine Veränderung erreicht werden könnte, machen die Vertre- 
tenden der beiden Unternehmen folgende Empfehlungen: An erster Stelle müsse das Schweizer System so umgestaltet werden, dass Geschlechtergleichheit gefördert wird und keinen Verlust für die Unternehmen darstellt. Dies könnte beispielsweise durch das Einführen eines Vaterschaftsurlaubs gelingen. Obwohl eine Frauenquote generell nicht befürwortet wird, könnte sie dann eine Lösung darstellen, wenn Freiwilligkeit zu keiner Veränderung in Unternehmen führt. Gute und bezahlbare Kinderkrippen sowie eine Individualbesteuerung können weitere wichtige Pfeiler sein, welche die Stellung der Frau auf dem Arbeitsmarkt weiter stärken. Letzteres wurde in Schweden bereits in den 1970er Jahren eingeführt; dies, da eine gemeinsame Besteuerung von Ehepartnern ein Hindernis für die Geschlechtergleichstellung bedeutete (Engcrantz sowie Westman im Interview; Martinek 2006: 15).

Eine Eigenschaft, welche beide schwedischen Unternehmen teilen, ist die lösungsorientierte Einstellung zum Umgang mit Schwierigkeiten. In Kungsleden würden Probleme adressiert und in Lösungen umgewandelt, erklärt Westman, und Engcrantz bringt es auf den Punkt: „Es ist dann ein Problem, wenn man es als Problem sieht".

\section{„Anti-Diskriminierung systematisch und analytisch angehen"}

Am 14. September 2017 stand der Besuch beim Equality Ombudsman (nachfolgend: DO) in der an Stockholm angrenzenden Gemeinde Solna an. Dort wurden Kerstin Jansson, internationale Koordinatorin, und Arja Lehto, Analystin, interviewt. Diese beiden gehören zu einem Team von rund 90 Mitarbeitenden, an dessen Spitze der „Ombudsman“ - eigentlich die Ombudsfrau - Agneta Broberg steht. Diese Behörde ist Bestandteil des Ministeriums für Gesund-

heit und Soziale Ordnung und für alle Formen der Diskriminierung zuständig (Europäisches Parlament 2015: 12).

Diese staatliche Beschwerdestelle existiert seit Anfang 2009 und ersetzt die vier bisherigen „Anti-Discrimination-Ombudsmen“. Ihr Mandat, also die Bekämpfung von Diskriminierungen aufgrund des Geschlechts, der sexuellen Orientierung, einer Transgender-Identität, der ethnischen Herkunft, einer Behinderung, der Religion und des Glaubens oder des Alters, erhält sie durch den vom Parlament im Jahre 2008 erlassenen „Discrimination Act“ (Government Offices of Sweden 2017).

\section{„Es ist ein Prozess, kein Dokument"}

Ein innovatives Element stellt der für alle Unternehmen obligatorische Förderplan für Chancengleichheit dar. Hierbei gilt es, jährlich den Massnahmenplan zur Förderung bei Arbeitsbedingungen, Lohngleichheit, Rekrutierung, Zugang zu Aus- und Weiterbildung, Kombinierung von Arbeit mit Elternschaft, Prävention (sexueller) Nötigung und zur gleichmässigen Verteilung des Geschlechts bei Arbeitnehmenden zu erstellen. Diese Berichte, in denen die erwähnten Aspekte einzeln begründet werden müssen, werden zuhanden des DO erstellt, die Verantwortlichkeit dafür liegt jedoch bei den Unternehmen. Der DO kann nicht alle Berichte kontrollieren, daher liegt der Fokus jeweils auf einer Branche oder einem Sektor, meist, wenn zuvor „Unregelmässigkeiten“ bekannt wurden. Ziel wäre es, dass diese Pläne nicht nur auf Papier niedergeschrieben sind, sondern in der täglichen Arbeit der Unternehmen gelebt wer- 
den. Um diesem Bestreben noch näher zu kommen, wäre es nach Aussage von Jansson denkbar, dass die DO die Unternehmen nicht mehr zu sich einlädt, sondern vor Ort besucht und an deren Wirkungsstätten die Umsetzung kontrolliert.

\section{Prävention und Analyse}

Zudem haben Bürgerinnen und Bürger im Falle von Diskriminierung die Möglichkeit, sich direkt an die DO zu wenden. Jährlich gehen laut Aussage von Letho ungefähr 2000 Individualbeschwerden ein, davon werden etwa 200 genauer studiert. $\mathrm{Zu}$ guter Letzt werden dann rund 20 Beschwerden daraus von der DO vor Gericht gebracht. Auswahlkriterium für die Fälle, welche vor Gericht gebracht werden, stellten der Vorbildcharakter und die Erfolgschancen dar. Bei den restlichen Beschwerden werde proaktiv gearbeitet, wie Letho ausführt, sprich, im Rahmen der präventiven Tätigkeit der schlichtende Dialog mit den involvierten Unternehmen oder Parteien gesucht.

Der Schwerpunkt liegt gemäss Letho jedoch weniger auf der Ebene der individuellen, sondern mehr auf jener der strukturellen Diskriminierung. So werden die eingehenden Individualbeschwerden vor allem auch statistisch aufgearbeitet und analysiert. Es soll möglichst alles bei der DO von einer strategischen Perspektive angegangen werden. Eigens dafür hat die DO eine ganze Analyseabteilung, zu der auch die Interviewpartnerin gehört. Jährlich werden dort beispielsweise Beschwerdestatistiken erhoben. 2016 und auch die Jahre zuvor seien demnach Diskriminierungen aufgrund von Behinderungen oder der ethnischen Herkunft die am meisten ausgeprägten Diskriminierungsformen gewesen. Davon hätten mehr als ein Drittel das Berufsleben betroffen. Im aktuellen Zeitraum richtet sich die Aufmerksamkeit auf die Themen Arbeitsleben und Unterbringung. Weiter soll - so Letho - zukünftig das Augenmerk auf Antidiskriminierung sowie auf gute Arbeitsbedingungen in Sozialversicherungen und in Sozialdiensten gerichtet werden.

\section{Der Vergleich zur Schweiz}

Bei der DO handelt es sich um eine nationale Stelle, die für alle Bürgerinnen und Bürger bei Diskriminierung als Anlaufstelle gilt. Etwas Vergleichbares existiert in der Schweiz nicht. Die Stelle leistet insbesondere durch ihre ausgeprägte Analyse- und Befragungstätigkeit auf nationaler Ebene, die auch immer wieder neue strategische Wege aufzeigt, einen grossen Beitrag zur Antidiskriminierung. Entsprechende Statistiken sind in der Schweiz im Vergleich nur mangelhaft vorhanden. Eindrücklich erscheint - aus Schweizer Perspektive - auch, dass das Erstellen dieser Unternehmensberichte auf wenig Widerstand bei den Firmen stösst (vgl. Ausführungen weiter oben und nachfolgend).

Übrigens blieb die männliche Namensgebung „ombutsman“ dieser Behörde nicht undiskutiert. Wie Jansson ausführt, ist man sich bewusst, dass hier ein Widerspruch vorhanden ist. Man habe jedoch die erlangte Bekanntheit nicht aufs Spiel setzen wollen und man sehe weniger die Semantik als die Funktionalität des Wortes „ombutsman“ im Vordergrund. 


\section{„Kinder haben ein Recht auf beide Elternteile“}

In Ergänzung zu Literatur und ExpertInneninterviews sollten Interviews auf den Strassen Stockholms einen Einblick ins Erleben der Familien- und Gleichstellungspolitik durch die schwedische Bevölkerung bieten. Dabei standen die Fragen im Zentrum, was die schwedische Bevölkerung an deren Familienpolitik schätzt, welche innovativen Elemente und Herausforderungen, aber auch Widersprüche sie erkennen und welche Visionen sie für die schwedische Familienpolitik haben. $\mathrm{Zu}$ zweit und zu dritt wurden durch die Studierenden und Dozierenden insgesamt 25 Strasseninterviews mit Müttern und Vätern durchgeführt. Die Eltern wurden in der Regel am Kinderwagen erkannt oder auf Kinderspielplätzen angesprochen. Dementsprechend sind die Befragten allesamt Eltern von mindestens einem Kleinkind und somit aktuell vom Elternurlaub betroffen. Die InterviewpartnerInnen waren im Alter zwischen 30 und 45 Jahren. Es wurden in etwa gleich viele Mütter wie Väter befragt. Die Interviews dauerten zwischen zehn und zwanzig Minuten und wurden in englischer Sprache geführt. Unter den Interviewten befanden sich sowohl Eltern mit ausschliesslich schwedischer Abstammung als auch solche mit Migrationshintergrund. Bis auf zwei Interviews wurden diese mit in Stockholm wohnhaften Personen geführt. ${ }^{6}$ Interviewt wurde in Strassen und auf Plätzen in Södermalm (einem Trendquartier im Stadtzentrum) und in Vorstadtquartieren der Botkyrka Kommun (Quartiere mit dem höchsten Migrationsanteil in Schweden).

Die schwedische Familienpolitik geniesst unter den Befragten eine breite Zustimmung. Sie wird als innovativ und fortschrittlich beschrieben, einige bezeichneten das System gar als das beste der Welt. Namentlich wurde hervorgehoben, dass in Schweden die väterliche Familienarbeit zur Norm gehöre, was dazu führe, dass häufig beide Elternteile arbeiten können. Gleichzeitig erlaubt dieses System auch beiden Elternteilen, sich aktiv an der Familien- und Erziehungsarbeit zu beteiligen. Es wurde auch aus der Perspektive der Kinder argumentiert, dass diese doch ein Anrecht auf beide Elternteile hätten. Es bestünde eine hohe Akzeptanz auf Seiten der Arbeitgebenden, Probleme bei der Umsetzung gäbe es nur sehr selten. Einige würde den gesetzlich vorgeschriebenen Lohn während des Elternurlaubs gar von 80 auf 90 Prozent erhöhen, dies wohl hauptsächlich aus Überlegungen in Zusammenhang mit einer langfristigen Mitarbeitendenbindung. Eine Interviewpartnerin erzählte, ihr Kind sei schwer erkrankt und es komme deshalb zu vielen Absenzen von ihr als Arbeitnehmerin. Auch hier gäbe es absolut kein Problem. Des Weiteren teilten Befragte mit, es fände eine Entlastung des erweiterten Familiensystems (Grosseltern etc.) statt, von welchen keine Übernahme der Betreuungsaufgaben erwartet würde.

\section{„Middle-Class"-Elternurlaub}

Eine Herausforderung des Systems ergibt sich womöglich aus der Tatsache, dass sich die Lohnfortzahlung während des Elternurlaubs am letzten Arbeitslohn der Bezügerinnen und Bezüger orientiert. So scheint es möglich, dass jüngere Paare mit der Erfüllung ihres Kinder-

\footnotetext{
${ }^{6}$ Bei den zwei Ausnahmen handelte es sich zum einen um einen Vater, welcher sich zu Ferienzwecken in Stockholm aufhielt. Zum anderen wurde ein Interview über Facebook mit einer schwedischen Bekannten eines Gruppenmitgliedes geführt.
} 
wunsches zuwarten, bis sich zuerst eine stabile Berufssituation eingestellt hat und sie dadurch besser entschädigt werden. Ob diese von den Interviewpartnerinnen und -partnern erwähnten Bedenken begründet sind, konnte nicht abschliessend überprüft werden. ${ }^{7}$ Weitere Herausforderungen wurden bezüglich der Zielgruppe erwähnt. Studenten und Studentinnen, Personen ohne permanentes Einkommen, sehr Vermögende oder aber auch arme Menschen würden nicht immer angemessen begünstigt werden. So gäbe es auf der einen Seite Maximalbeträge für den versicherten Lohn (was zu Kritik von viel Verdienenden führt, die an einen Plafond stossen), auf der anderen Seite sei das allgemeine Familiengeld sehr tief (was die unteren Schichten bezüglich Einkommen kritisieren). Das System wurde daher teilweise als „MiddleClass-Thing" bezeichnet.

Die Befragten skizzierten schliesslich einige Verbesserungsvorschläge. So wurde angemerkt, dass sich die Höhe des Elterngeldes nicht ausschlich auf den letzten Lohn beziehen solle. Es müsse eine Formel zur Berechnung gefunden werden, die den realen Ansprüchen besser gerecht werde als derzeit. Einige weitere Äusserungen bezogen sich auf Schwierigkeiten mit ausländischen Firmen in Schweden. Es wurde berichtet, dass hier noch Handlungsbedarf bestünde, dass auch diese das System vollständig akzeptieren und umsetzen sollten. Weiter wurde kritisiert, dass durch die hohe Arbeitstätigkeit der Eltern der Kontakt zu deren Kindern zu kurz komme. Die Kinder hätten ein Anrecht darauf, mit ihren Eltern mehr Zeit zu verbringen und die Beziehung zu ihnen intensiver zu erleben. Die Gesellschaft erwarte mittlerweile, dass immer beide Elternteile arbeiten würden. Ein bewusster Entscheid für die Rolle als Hausfrau oder -mann, ohne Erwerb generierende Tätigkeit, stosse in der heutigen Zeit teilweise auf Unverständnis. Auch wenn das schwedische System in vielen Bereichen vorbildlich sei, bestünde in den genannten Bereichen Handlungsbedarf, es müsse stetig thematisiert und weiterentwickelt werden.

\section{"Gendersensitivität als Gewaltprävention“}

Am 13. September 2017 stand der Besuch der sozialen Dienste der Gemeinde Botkyrka (nahe Stockholm) in der Ortschaft Tumba an. Das ExpertInneninterview vor Ort wurde mit Robin Glad und Mantha Kasagianni von der Präventionseinheit der sozialen Dienste geführt. Im Zentrum des Interviews stand die Frage, ob im Beratungskontext geschlechterspezifische Rollenerwartungen bestehen. Es interessierte einerseits, ob die sozialen Dienste Frauen und Männer unterschiedlich adressieren und andererseits, ob und wie die Dimension Gender spezifische Aufmerksamkeit erhält.

Die Tätigkeitsgebiete der Präventionseinheit von Botkyrka umfassen gemäss den beiden Interviewten die Prävention an öffentlichen Vorschulen, Unterstützung von Familien, Unterstützung bei häuslicher Gewalt und Kindsmissbrauch, Unterstützung von Jugendlichen sowie Timeouts für Kinder mit Verhaltensauffälligkeiten inklusive Unterstützung deren Familien.

\footnotetext{
${ }^{7}$ Ein Hinweis könnte im zunehmenden Alter der schwedischen Mütter bei der Geburt ihres ersten Kindes liegen. Sie bekamen 2015 im Durchschnitt ihr erstes Kind im Alter von 29.2 Jahren; 1995 lag dieses Durchschnittsalter noch bei 27.9 Jahren (OECD 2017b). Dieser Altersanstieg hat noch weitere Gründe und der Trend zeigt sich auch in anderen europäischen Ländern. In der Schweiz lag der Altersdurchschnitt der Mütter bei der Erstgeburt im Jahr 2015 bei 30.6 Jahren, während er 2000 noch 28.7 Jahre betrug (OECD 2017).
} 
Robin Glad und Mantha Kasagianni sind insbesondere auf ihre Angebote im Bereich der Gewaltprävention eingegangen. Der angewendete Ansatz zielt auf die Förderung von Zivilcourage und stellt gängige Geschlechterstereotypen in Frage.

Ausgangslage ist, dass in Botkyrka einst die Problematik der Gewalt an Schulen zumindest für Jungen zur Normalität geworden ist. Gemäss Glad und Kasagianni gibt es einen Zusammenhang zwischen Geschlechterstereotypen und Anwendung von Gewalt. So sind junge Männer 4.4 mal stärker gefährdet, gewalttätig zu sein, als junge Frauen. Eine Umfrage mit 377 Schülerinnen und Schülern (4-11 Jahre) in Botkyrka hat aufgezeigt, dass bereits in jungen Jahren klare geschlechterspezifische Erwartungen vorhanden sind. Deshalb ist eine Gendersensitivität, die bereits durch die Eltern und später durch die Grundschule vermittelt wird, ein wichtiges präventives Hilfsmittel, um Gewalt zu verhindern. Damit diese Gendersensitivität und die Gewaltprävention möglichst nachhaltig umgesetzt werden können, ist es die Aufgabe der Sozialen Arbeit, einerseits die Professionellen (Lehrpersonen, Polizei) und andererseits die Schülerinnen und Schüler, sowie ihre Eltern zu sensibilisieren und zu schulen. Dieser Umstand sollte seitens der Präventionseinheit adressiert werden. In der Folge wurde der MVPAnsatz (mentors in violence prevention) nach Jackson Katz implementiert (vgl. MVP Strategies 2017). Es handelt sich dabei um einen „bystander approach“. Das heisst, es sollen bei einem Übergriff unbeteiligte, aber anwesende Leute (hier Kinder) dazu befähigt werden, adäquat in einer Situation zu intervenieren. Der Ansatz umfasst ebenfalls, dass eine generelle Kultur der Toleranz gefördert wird und Normen, welche zu Gewalt führen, als inakzeptabel dargestellt werden.

Aus der Literatur ist der Begriff des doing gender bekannt. Gemeint ist damit die alltägliche Praxis sozialer Interaktionen, in welchen die Bedeutungen und Definitionen von Geschlecht rekonstruiert werden (Brückner 2002: 270). Der MVP-Ansatz trägt der Erkenntnis Rechnung, dass bestimmte (männliche) Geschlechterstereotypen die Ausübung von Übergriffen begünstigen. In Botkyrka werden mittels des MVP-Ansatzes die sozialen Praxen und Stereotypen problematisiert, welcher einer Gleichstellung der Geschlechter entgegenstehen. Die methodische Umsetzung des MVP-Ansatzes in Botkyrka sieht so aus, dass Schülerinnen und Schüler ab zwölf Jahren (6. Klasse) eine Mentorin oder einen Mentor aus der neunten Klasse zugeteilt bekommen. Die Mentorinnen und Mentoren sprechen mit den jüngeren Kindern über Gewalt und diskriminierende (männliche) Normen. Die MVP-Mentorinnen und -Mentoren wiederum werden durch Coaches betreut, welche z.B. Lehrpersonen sind, die im MVP-Ansatz geschult sind. Eine besonders innovative Leistung der Präventionseinheit in Botkyrka ist laut den Interviewten diejenige, dass der MVP-Ansatz weiterentwickelt wurde und unterdessen in angepasster Form bereits bei Kindern ab sechs Jahren zum Einsatz kommt.

Die professionelle Soziale Arbeit ist bei ihren Interventionen grundsätzlich mit dem Risiko behaftet, Geschlechterdiskriminierungen zu reproduzieren, wie dies auch in anderen Gesellschaftsbereichen der Fall ist. Angesprochen auf die Frage, wie in Botkyrka dieser Problematik auf professioneller Ebene begegnet wird, gaben Glad und Kasagianni zur Auskunft, dass Gendersensitivität mehr eine Frage der Haltung als die eines Instruments sei. Auch Ehlert (2012) argumentiert dahingehend, dass Professionelle der Sozialen Arbeit eine reflexive Grundhaltung einzunehmen hätten, was dann zu einer geschlechter- bzw. diversitätsbewuss- 
ten Sozialen Arbeit führe (Ehlert 2012: 108). Der Aspekt der Gendersensitivität findet in der Ausbildung der Sozialen Arbeit in Schweden gemäss Glad und Kasagianni grossen Eingang.

\section{Herausforderungen und Zukunftspläne}

Botkyrka hat viel kostengünstigen Wohnraum und ist gleichzeitig ländliches Gebiet. Die Wohnbevölkerung ist sehr multikulturell und divers (Botkyrka Kommun 2017). Gemäss Glad und Kasagianni ist es eine Herausforderung, die Zielgruppe des MVP-Projektes so zu adressieren, dass sie das Projekt wahrnehmen. Um möglichst viele Einwohnerinnen und Einwohner von Botkyrka ansprechen zu können, muss die Soziale Arbeit niederschwellige Wege finden. Vor allem bei Menschen mit schlechten schwedischen Sprachkenntnissen ist ein einfacher Zugang wichtig, da sonst Vorbehalte gegenüber Sozialdiensten gefestigt werden. Deshalb ist der Sozialdienst bei Elternabenden und in den Quartieren aufzufinden. Zudem ist er auf verschiedenen Kanälen wie den sozialen Medien aktiv. Das Ziel ist, dass die Soziale Arbeit Teil des Alltags in der Schule und eine zugängliche Anlaufstelle wird.

Gemäss Aussagen von Glad und Kasagianni kommt es regelmässig zu einer Reproduzierung der Geschlechterstereotypen durch Lehrpersonen; deshalb ist eine gezielte Schulung der Professionellen ein wichtiger Bestandteil des Projektes. Die verschiedenen Präventionsprojekte werden bis jetzt in ausgewählten Schulen umgesetzt, jedoch sollten - trotz Sparmassnahmen alle Schülerinnen und Schüler mit dem eigenen Rollenverständnis konfrontiert werden und in Gewaltprävention geschult werden.

\section{"Demokratie und Gleichstellung der Geschlechter sind nicht geschenkt"}

Ein letztes Expertinneninterview wurde am 15. September 2017 mit Brigitta Notlöf, Betriebsleiterin von Livstycket, durchgeführt. Livstycket ist eine Non-Profit-Organisation (NPO), die 1992 in Tensta in einem Vorort von Stockholm gegründet wurde. Sie ist heute ein modernes Knowledge and Design Centre und verfolgt als Hauptziel die soziale und kulturelle Integration von Migrantinnen. Die NPO wird von einem freiwilligen Vorstand geleitet, acht Personen wurden 2016 eingestellt, 384 Mitglieder umfasst die Organisation in ganz Schweden. 118 Migrantinnen aus der ganzen Welt haben letztes Jahr partizipiert (Notlöf 2016: 18).

Das Wort Livstycket heisst - gemäss Aussage von Notlöf - direkt übersetzt Korsage. Die (Winter-)Korsage wurde traditionell im Winter von Frauen, Männern und Kindern getragen und symbolisiert darum Wärme und Stütze. Schreibt man Livstycket in zwei Wörter, erhält es eine ganz andere Bedeutung, denn Liv bedeutet Leben und Stycket bedeutet Stück, was dann ein Stück Leben bedeutet.

Die NPO wird von der Stadt Stockholm, von diversen Spenden, dem Erlös des Verkaufs von Textilien und durch verschiedene Stiftungen finanziert (Notlöf 2016: 18). Das Herz von Livstyket ist die Betriebsleiterin Brigitta Notlöf. Sie startete vor über 25 Jahren mit dem Erlös eines Flohmarktes und dem Verkauf ihres Autos. Danach erhielt sie für ihr Projekt zwölf Quadratmeter Raum in einer Schule. Während des Interviews wird schnell klar, wer da steht: eine Frau der Tat, eine Kämpfernatur und dazu noch eine, die nach über 25 Jahren Arbeit für ihre NPO nicht frustriert ist. „Wir müssen kämpfen“, meint sie. Demokratie und Gleichstel- 
lung der Geschlechter, das sei etwas, was wir nicht geschenkt bekommen. Diese Kraft und positive Haltung von Brigitta Notlöf haben Livstyket geprägt.

\section{Praktisches, kreatives Lernen für Frauen}

Die schwedische Sprache ist laut Notlöf der Schlüssel, um ein Mitglied der Gesellschaft zu sein. Durch das Lernen der Sprache soll das Selbstwertgefühl der Teilnehmerinnen gesteigert und somit ihre Isolation durchbrochen werden. Der Grundsatz in Livstyket heisst darum auch Empowerment. Im Fokus stehen die Teilnehmerinnen und ihre Ressourcen (Livstyket 2017). Sie werden darum nach dem Schulniveau, welches sie in ihrem Heimatland hatten, aufgeteilt. Die Frauen werden dazu bemächtigt, ihr Humankapital auszuschöpfen. Dies wird in der Sprachschule mit funktionaler Fremdsprachendidaktik angestrebt. Dabei wird theoretisches und praktisches kreatives Lernen mit der Sprache verbunden. Jährlich werden sechs verschiedene Themen behandelt. Als erstes wird das gelernt, was den Frauen vertraut ist (z.B. Körper, Gesundheit und Nahrungsmittel), dann wird übergegangen zu unbekannteren Themen (öffentlicher Verkehr wie Untergrundbahnen und Tram, schwedische Kultur und Geschichte). Um das Gelernte in einen Kontext setzen zu können, werden nach dem dritten Thema Aktivitäten durchgeführt (etwa Museen besucht) und Textilwaren bestickt, bedruckt oder neue Muster entwickelt. Die Verbindung von Theorie und Praxis setzt die neu erlernten Wörter in eine Funktion und gibt ihnen so ihre Bedeutung (Notlöf 2016: 18).

Die kreativen Aktivitäten sind jedoch auch ein Weg, um Erinnerungen zu verarbeiten und Neues zu lernen. Die Designs entstehen demnach durch verschiedene Projekte. Die Frauen zeichnen, was sie sehen, fühlen und erfahren und somit steht hinter jedem Muster eine Geschichte. Das Muster the whole world in a bag erzählt z.B. die Geschichte von Menschen auf der Flucht, wie diese ihr ganzes Leben in einer Tasche verstauen und entscheiden müssen, was sie mitnehmen beziehungsweise hinter sich lassen (Aussage von Notlöf). Mit diesem Muster bedruckt lassen sich heute Taschen, Kulturbeutel und Kochschürzen kaufen.

\section{Migrations- und Integrationspolitik in Schweden - die grössten Herausforderungen}

Auf die Frage, was denn die grössten Probleme im Migrationsbereich in Schweden seien, antwortet Brigitta Notlöf: „Es sind einfach zu viele. Zu viele auf einmal!“ 2015 gab es in Schweden 163`000 Asylanträge (Parusel 2017). Im Vergleich dazu hatte die Schweiz 2015 nur rund 40`000 Anträge (Staatsekretariat für Migration 2016). Nach 2015 gab es in Schweden einen drastischen Rückgang der Anträge, dennoch herrscht eine Überforderung. Es gäbe zu wenig Wohnraum, meint Notlöf. Frustration, Isolation und Gewalt seien die Folgen.

Zum Schluss erwähnt Notlöf, dass der berühmte österreichische Architekt und Designer Josef Frank in der Zwischenkriegszeit als Flüchtling nach Schweden kam. Mit seinen Entwürfen trug er dazu bei, dass das Designunternehmen Svenskt Tenn zu einem der bekanntesten und wichtigsten Designerunternehmen in Schweden wurde. Die Entstehung dieses Kulturguts war gemäss Notlöf nur möglich durch die Offenheit der Schwedinnen und Schweden. In Brigitta Notlöfs Worten lässt sich die multikulturelle Haltung in Schweden heraushören. Die Integrationspolitik Schwedens ist eine der ehrgeizigsten in Europa. Der öffentliche Sektor sorgt für soziale Sicherheit für Ausländerinnen und Ausländer wie auch für Staatsbürgerinnen 
und Staatsbürger. Gleichheit der Rechte, Gerechtigkeit, Solidarität und Kooperation sind Kernwerte dieser Wohlfahrtsideologie. Auf der individuellen Ebene lässt sich in Schweden der Universalismus an der Chancengleichheit der Kinderbetreuung erkennen: Der Kindergarten und die Kinderkrippen sind ein Recht der Kinder. Die externe Kinderbetreuung hat zudem einen grossen Einfluss auf die Integration der Kinder (Hammar 2003: 238).

\section{Kinderrechtszentriertheit, Lösungsorientierung, Evidenzbasierung und gelebte Gleichstellung - ein vorläufiges Fazit}

Wir sind auf unsere Studien- und Forschungsreise gestartet mit dem Wissen um Schwedens Individualbesteuerung, gut ausgebaute, öffentliche Kinderbetreuung und grosszügige Elternurlaubsversicherung. Wir haben dazu in den ExpertInnen- und Strasseninterviews vertiefte Informationen erhalten und erfahren, wie zentral die genannten familienpolitischen Elemente aus den verschiedenen Perspektiven sind. Anders als aufgrund von Analysen aus der Schweiz erwartet (Lanfranconi 2014), wird dabei in Schweden weit weniger mit der Eingliederung der Frauen in den Arbeitsmarkt und dafür vielmehr mit dem Wohl und Recht der Kinder argumentiert. So wird ein von Vätern und Müttern bezogener Elternurlaub als eine dem Wohl der Kinder förderliche Massnahme eingestuft und die Möglichkeit für alle Kinder, eine Kita zu besuchen, als ein Recht des Kindes auf ausserhäusliche Kontakte betrachtet.

Ein weiterer Kern aller ExpertInneninterviews war zudem die positive, lösungsorientierte Haltung aller Fachleute und Institutionen. In den beiden besuchten Unternehmen beispielsweise wird der Blick darauf gerichtet, dass Gleichstellung und Diversity in den Führungsetagen sowie im Gesamtunternehmen ein Gewinn für das Unternehmen und dessen Erfolg sind. Sowie, dass Hürden, die dem im Weg stehen (wie z.B. hier in der Schweiz das Fehlen von Kita-Plätzen oder dem Vaterschaftsurlaub), abgebaut werden müssen. Unsere Erwartung betreffend die - im Vergleich zu der Schweiz - mit sehr vielen Ressourcen ausgestatteten Ombudsstelle war, dass diese Stelle v.a. Kontrollen von Unternehmen ausübt und Diskriminierung sanktioniert. Dies wird auch gemacht. $\mathrm{Zu}$ einem grossen Teil werden die Ressourcen aber auch für einen Dialog eingesetzt, um sich positiv in die Gleichstellungsprozesse in den Unternehmen einzumischen. Das gleichzeitige, gezielte sanktionieren von Diskriminierungsfällen mit Vorbildcharakter scheint eine gewinnbringende Strategie zu sein. In einem ganz anderen Bereich, im Frauenintegrationsprojekt Livstycket, wird ebenfalls klar ressourcen- und lösungsorientiert vorgegangen, indem an die bestehenden Ressourcen der Frauen angeknüpft wird und sie in der Bewältigung ihres Alltags ermächtigt werden.

Ebenfalls erstaunte die in diversen ExpertInneninterviews geäusserte starke Gewichtung wissenschaftlicher Befunde bei Entscheiden auf den verschiedenen Ebenen der Familien- und Gleichstellungspolitik. So schilderte Professorin Duvander eindrücklich, dass die „DaddyMonths" mehrmals verlängert worden seien, einfach, weil wissenschaftliche Studien aufgezeigt hätten, dass dieses Instrument wirksam sei. Die nationale Ombudsstelle ihrerseits fokussiert ihre Ressourcen auf die Themenfelder, wo laut ihren eigenen Analysen der grösste Handlungsbedarf besteht. Und auch die sozialarbeiterischen Projekte zur Gewaltprävention fussen scheinbar natürlich auf einer eigenen Analyse der Bilder und Stereotypen von Mädchen und Jungen. 
Eine weitere beeindruckende Erkenntnis aus all den Interviews ist der Umfang, in welchem Geschlechtergleichstellung in den Institutionen, Programmen und im Alltag gelebt und mitgedacht wird. Dies zeigt sich etwa daran, wie in schulischen Gewaltpräventionsprojekten ganz selbstverständlich zuerst einmal geschlechtsspezifische Rollenbilder thematisiert und zu durchbrechen versucht werden. Ob in der Forschung, der Wirtschaft, der Politik, oder auch in den Strasseninterviews, es zeigt sich; Geschlechtergleichstellung und Chancengleichheit sind klare Ziele, aber v.a. auch gelebte Realitäten, welche sich auch in Bildern und Symbolen im öffentlichen Raum ausdrückt (vgl. Abb. 1).

\section{Abbildung 1: Aufschrift auf einer Unisex-Toilette}

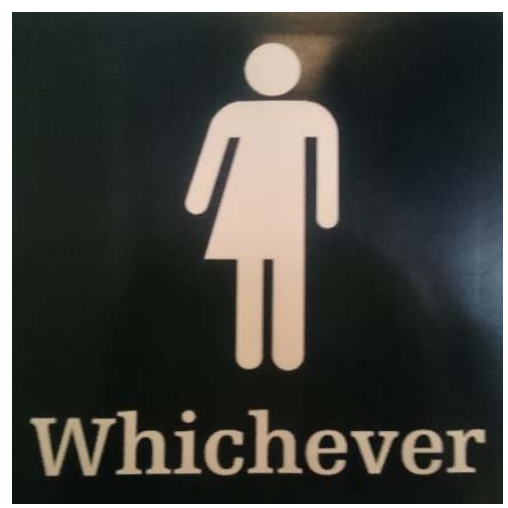

Quelle: Lucia M. Lanfranconi

\section{Zusammenfassend: Herausforderungen und Lösungsvorschläge}

Dennoch, auch die Gleichstellungs- und Familienpolitik Schwedens ist nicht perfekt. Zentrale in den Interviews angesprochene Herausforderungen und Lösungsvorschläge sind die Folgenden:

- Es gibt in Schweden eine gesellschaftliche Norm, (zu einem hohen Prozentsatz) zu arbeiten. Inwiefern kann da von Wahlfreiheit für Mütter und Väter gesprochen werden?

- Frauen werden nach wie vor - wenn auch in weitaus kleinerem Masse als in der Schweiz - z.B. durch tiefere Löhne auf dem Arbeitsmarkt benachteiligt und sind immer noch mehrheitlich für die Kinderbetreuung und -erziehung verantwortlich.

- Umgekehrt beziehen in Schweden die Väter noch den tieferen Anteil des Elternurlaubes und kümmern sich etwas weniger um die Kinder als die Mütter. Dem könnte laut den Interviews Abhilfe geleistet werden, indem z.B. der Anteil des Elternurlaubes, der vom Vater bezogen werden muss („Daddy-Months“), nochmals erhöht würde. Zudem bräuchte es noch bessere Möglichkeiten für Männer (in der Privatwirtschaft), Teilzeit $\mathrm{zu}$ arbeiten und Elternurlaub auch in Kaderfunktionen beziehen zu können.

- Eine weitere mögliche Massnahme zur Vorbeugung von Diskriminierung wäre, Bewerbungsunterlagen ohne Angaben des Geschlechtes, des Alters oder der Herkunft einzuführen, sodass effektiv nur auf die Kompetenzen einer Person geachtet wird.

- Die Lohnfortzahlung während des Elternurlaubs orientiert sich am letzten Arbeitslohn der Bezüger und Bezügerinnen. So scheint es ein Problem, dass Paare mit dem Kinder- 
kriegen zuwarten müssen, bis sich eine stabile Berufssituation eingestellt hat und sie dadurch besser entschädigt werden.

- Studenten und Studentinnen, Personen ohne permanentes Einkommen, sehr Vermögende oder aber auch arme Menschen werden nicht immer angemessen begünstigt vom Elternurlaub, so eine weitere Kritik. So gebe es auf der einen Seite Maximalbeträge für den versicherten Lohn (was zu Kritik von viel Verdienenden führt), auf der anderen Seite sei das allgemeine Familiengeld sehr tief (was die unteren Schichten bezüglich Einkommen kritisieren). Das System wird in den Interviews teilweise als „MiddleClass-Thing“ bezeichnet. Es müsse eine Formel zur Berechnung gefunden werden, die den realen Ansprüchen besser gerecht werde.

- Ausländische Firmen in Schweden akzeptieren gemäss Interview-Aussagen das System teilweise nicht und setzen es entsprechend unvollständig um.

- Reproduzierungen von Geschlechterstereotypen durch Lehrpersonen, Eltern und Kinder sind in Schweden nach wie vor ein Problem. Daher sind gezielte Schulung und konkrete Projekte noch immer nötig.

- Auch in Schweden gibt es im Bereich der Sozialpolitik diverse Sparbemühungen. So müssen beispielsweise Präventionsprojekte und Integrationsprojekte für Flüchtlinge immer wieder neu legitimiert werden. Anders als in der Schweiz scheint jedoch die Familien- und Gleichstellungspolitik weniger von solchen Sparbemühungen betroffen, da sie stärker in der Gesellschaft verankert sind und alle von ihnen profitieren, und weil ein entsprechendes Bewusstsein bei allen AkteurInnen vorhanden ist.

\section{Vorbild für die Schweiz?}

Schwedens kohärente, umfassende und gelebte Gleichstellungs- und Familienpolitik ist definitiv ein Vorbild für die Schweiz. Dies kommt klar aus den unterschiedlichen Interviews und Diskussionen heraus. In der Schweiz gibt es zahlreiche Hürden für die Implementierung vergleichbarer Politiken und Massnahmen. So etwa behindern und verzögern tendenziell Föderalismus und direkte Demokratie wie auch eine Mitte-Rechts-Mehrheit in Parlament und Bundesrat zusätzliche Ausgaben im Bereich der Sozialpolitik im Generellen und der Familienund Gleichstellungpolitik im Besondern (Armingeon 2001; Lanfranconi/Valarino 2014; Trampusch/Mach 2011). Weitere spezifische Hürden sind das Fehlen einer gesetzlichen Basis für eine nationale Familienpolitik, ${ }^{8}$ das bisherige Fehlen jeglicher Vaterschafts- oder Elternurlaube (Bundesrat 2017), die nach wie vor mangelnden und zu teuren Kinderbetreuungseinrichtungen, ${ }^{9}$ sowie das bisherige Fehlen von Durchsetzungsmechanismen im Bereich der Gleichstellungspolitik. ${ }^{10}$ Gerade das Fehlen solcher Massnahmen macht den Handlungsbedarf in der Schweiz deutlich. So würden diese Massnahmen sowohl zum Wohl der Kinder und der Gleichstellung von Väter und Mütter resp. Frauen und Männern als auch zum Gedeihen der

\footnotetext{
${ }^{8}$ So ist der „Familienartikel“ in der Verfassung 2013 sehr knapp am Ständemehr gescheitert (Bundesamt für Sozialversicherung 2017a).

${ }^{9}$ Wobei das Problem erkannt ist und seit Jahren Anschubfinanzierungen zur Schaffung weiterer Kitas laufen (Bundesamt für Sozialversicherungen 2017b).

${ }^{10}$ Mögliche solcher Massnahmen wären etwa obligatorische Lohnkontrollen, Gleichstellungspläne oder ähnliches (Lanfranconi et. al. 2017).
} 
Wirtschaft beitragen. Um mit einem Zitat aus dem Interview mit Frau Ylva Sarby Westman (Deputy CEO in Kungsleden) zu enden: „Sterben die Unternehmen bei euch in der Schweiz, welche nicht auf Gleichstellung und Diversity setzen, nicht aus?“

\section{Literaturverzeichnis}

AllBright (2016). One in five executives are women. The AllBright Report, March 2016. Internet-Site. Zugriff am 01.10.2017 auf https://static1.squarespace.com/static/5501a836e4b0472e6124f984 /t/587ca50fcd0f68a2e5dd7ef4/1484563733811/AllBrightrapporten+2016 ENG.pdf.

Armingeon, Klaus (2001). Institutionalising the Swiss Welfare State. West European Politics, 24(2), 145-168.

Botkyrka Kommun (2017). Botkyrka. Internet-Site. Zugriff am 20.08.2017 auf https://www.informationsverige.se/Engelska/Leva-och-bo/lan-och-kommuner/Pages/Botkyrka. aspx.

Brückner, Margrit (2002). On social work and what gender has got to do with it. European Journal of Social Work, 5(3), 269-276.

Bundesamt für Sozialversicherungen (2017b). Bericht zur Familienpolitik. Internet-Site. Zugriff am 15.12.2017 auf https://www.bsv.admin.ch/bsv/de/home/sozialpolitische-themen/familienpolitik/ grundlagen/tornare.html.

Bundesamt für Sozialversicherungen (2017b). Familienergänzende Kinderbetreuung. Internet-Site. Zugriff am 15.12.2017 auf https://www.bsv.admin.ch/bsv/de/home/finanzhilfen/kinderbetreuung html.

Bundesrat (2017). Bundesrat lehnt Volksinitiative für einen Vaterschaftsurlaub ab. Medienmitteilung des Bundesrates. Online-Mitteilung. Internet-Site. Zugriff am 19.12.2017 auf https://www.admin.ch/gov/de/start/dokumentation/medienmitteilungen.msg-id-68457.html.

Bundesrat (2015). Vollkosten und Finanzierung von Krippenplätzen im Ländervergleich. Bericht des Bundesrates. Bern: Bundesrat.

Duvander, Ann-Zofie und Gunnar Andersson (2006). Gender Equality and Fertility in Sweden. Marriage \& Family Review, 39, 121-142.

Duvander, Ann-Zofie und Tommy Ferrarini (2013). Sweden's Family Policy under Change: Past, Present, Future. Stockholm: Friedrich-Ebert-Stiftung.

Ehlert, Gudrun (2012). Gender in der Sozialen Arbeit. Konzepte, Perspektiven, Basiswissen. Schwalbach: Wochenschau Verlag.

Esping-Andersen, Gøsta (1990). The three worlds of welfare capitalism. Cambridge: Polity Press.

Europäisches Parlament (2015). The Policy on Gender Equality in Sweden. Brüssel: Europäisches Parlament.

Eurostat (2017). Datenbank. Formal childcare by age group and duration-\% over the population of each age group - EU-SILC survey. Zugriff am 02.01.2018 auf http://appsso.eurostat.ec.europa. $\underline{\text { eu/nui/show.do?dataset=ilc caindformal\&lang=en. }}$ 
Eurostat (2015). Datenbank. Europäische Statistik. Frauen in der EU sind bei der Geburt ihres ersten Kindes durchschnittlich fast 29 Jahre alt. Zugriff am 19.12.2017 auf http://ec.europa.eu/eurostat /documents/2995521/6829224/3-13052015-CP-DE.pdf/688d65c6-030a-4209-9393-eeb9a587ffba.

Government Offices of Sweden (2017). The Equality Ombudsman (DO). Internet-Site. Zugriff am 21.08.2017 auf http://www.government.se/government-agencies/equality-ombudsman-do/.

Hammar, Thomas (2003). Einwanderung in einen skandinavischen Wohlfahrtsstaat. Migration im Spannungsfeld von Globalisierung und Nationalstaat, 22, 225-252.

Häusermann, Silja (2006). Kinderbetreuung im internationalen Vergleich. Vortrag auf der Fachtagung „Umfassende Kinderbetreuung - Modelle für die Schweiz“, 18. September 2006, Volkshaus, Zürich. Präsentation. Zugriff am 19.12.2017 auf https://www.gleichstellung.uzh.ch/dam/jcr:0000000042f2-8a53-ffff-ffffd897b666/publication_225.pdf.

Jönsson, Ingrid (2002). Vereinbarkeit von Berufs- und Familienleben in Schweden. WSI Mitteilungen, 3/2002, 176-183.

Lanfranconi, Lucia M. (2014). Geschlechtergleichstellung durch Wirtschaftsnutzendiskurs? Dissertation. Universität Fribourg und FernUniversität in Hagen, Fribourg und Hagen. Zugriff am 15.12.2017 auf https://doc.rero.ch/record/234259?!n=de.

Lanfranconi, Lucia M., Gesine Fuchs, Marianne Müller und Sarah Rabhi-Sidler (2017). Gleichstellungspolitik in der Schweiz. E-Learning-Text. Zugriff am 01.10.2017 auf http://gleichstellen.ch /src/media/gleichstellen-ch-Hintergrundinfos-Gleichstellungspolitik.pdf.

Lanfranconi, Lucia M. und Romana Lanfranconi (2016). Gleichstellen - eine Momentaufnahme. Dokumentarfilm. Zugriff am 21.11.2017 auf http://gleichstellen.ch/film.

Lanfranconi, Lucia M. and Isabel Valarino (2014). Gender Equality and Parental Leave Policies in Switzerland. A Feminist and Discursive Perspective. Critical Social Policy, 34(4), 1-24.

Livstyket (2017). This is Livstyket. Internet-Site. Zugriff am 02.10.2017 auf http://www.livstycket. com/03.foreningen/detta_ar_eng.htm.

Martinek, Hanne (2006). Schweden: Vorbild für die Förderung individueller Existenzsicherung von Frauen. Working Papers des Projekts „Ernährermodell“ FB Politik-und Sozialwissenschaften der FU Berlin. Berlin: Freie Universität Berlin.

MISSOC - Mutual Information System on Social Protection (o.J.). MISSOC Comparative Tables Database. Online-Datenbank. Zugriff am 01.10.2017 auf http://www.missoc.org/MISSOC/INFORMATIONBASE/COMPARATIVETABLES/MISSOCDAT ABASE/comparativeTableSearch.jsp.

Müller, Marianne, Lucia M. Lanfranconi, Gesine Fuchs und Sarah Rabhi-Sidler (2017). Gleichstellung im Bereich der Elternschaft in der Schweiz. E-Learning-Text. Zugriff am 02.01.2018 2017 auf http://gleichstellen.ch/src/media/gleichstellen-ch-Hintergrundinfos-Elternschaft.pdf.

MVP Strategies (2017). Mentors in Violence Prevention. Mvpstrat. Internet-Site. Zugriff am 30.09.2017 auf http://www.mvpstrat.com/.

Notlöf, Brigitta (2016). Livstycket 2016 Business Report. Stockholm: Livstycket.

OECD (2017a), Familiy Database. Internet-Site. Zugriff am 21.12.2017 auf http://www.oecd.org/els/ family/LMF_1_2_Maternal_Employment.pdf. 
OECD (2017b). Familiy Database. Internet-Site. Zugriff am 21.12.2017 auf http://www.oecd.org lels/soc/SF 2 3 Age mothers childbirth.pdf.

OECD (2017c). OECD Economic Surveys. Sweden. Internet-Site. Zugriff am 02.01.2018 auf https://www.oecd.org/eco/surveys/Sweden-2017-OECD-economic-survey-overview.pdf.

OECD (2016). Time use for work, care and other day-to-day activities. Internet-Site. Zugriff am 02.01.2018 auf http://www.oecd.org/els/family/LMF2 5 Time use_of_work_and_care.pdf.

Parusel, Bernd (2017). Asylpolitische Restriktionen und ihre Folgen am Beispiel Schweden. Internet-Site. Zugriff am 02.10.2017 auf http://fluechtlingsforschung.net/asylpolitische-restriktionen-und-ihrefolgen-am-beispiel-schweden/.

Schär Moser, Marianne und Silvia Strub (2011). Massnahmen und Instrumente zur Bekämpfung der geschlechtsspezifischen Lohndiskriminierung. Die Schweiz im Spiegel des europäischen Auslands. Dossier 79. Bern: Schweizerischer Gewerkschaftsbund.

Staatsekretariat für Migration (2016). Asylstatistik 2015. Internet-Site. Zugriff am 02.01.2018 auf https://www.sem.admin.ch/sem/de/home/aktuell/news/2016/2016-01-28.html.

Trampusch, Christine und André Mach (2011). The Swiss Political Economy in Comparative Perspective. In: Trampusch, Christine und André Mach (Hg.). Switzerland in Europe: Continuity and Change in the Swiss Political Economy. Abingdon: Routledge, 11-26. 